\title{
Laser synthesis of optical media with silver nanoparticles by nanosecond pulses at
}

\author{
air. \\ V.K. Goncharov, K.V. Kozadaev, D.V. Shchehrykovich \\ A.N.Sevchenko Scientific-Research Institute of Applied Physical Problems of Belarussian State University, 7 \\ Kurchatov Str., 220108, Minsk, Belarus, kozadaeff@mail.ru
}

\begin{abstract}
The present work is dedicated to further development of the laser synthesis method for metal nanoparticles formation at air conditions. On the base of complex investigation of processes taking place during laser erosion of metals by intensive nanosecond pulses the method of silver nanoparticles fluxes is proposed. By the implantation of silver nanoparticles into liquid media (water or solutions of a polymeric base) the transarent media with silver nanoparticles can be obtained (particularly water colloids and modified polimer films). For the aims of investigation of formed polymeric films the complex diagnostics method is used
\end{abstract}

Key words: laser erosion of metals, silver nanoparticles, transmission electron microscopy, absorption spectroscopy, surface plasmon resonance.

\section{Introduction}

Nowadays the range of application of nanotechnologies is extremely diverse: it spreads from pharmaceutics and medicine to the applications in electronic and optical industry. The advantages of nanoobjects are caused by the appearance of size effects which allows to present exotic features in nanostructured materials which is not peculiar to their massive analogs. In connection with that the development of nanosized objects formation technologies is of considerable practical interest.

The production of isolated metal particles is particularly one of the most dynamically emerging sectors of nanotechnology. The number of industrial technologies based on physical and chemical principles is currently devised for the formation of metal nanosized objects [1-5]. Furthermore, the laser induced synthesis of nanosized objects under the ablation of monometallic targets is one of the most promising methods of the formation of nanosized metal objects [6]. The above-noted method possesses high efficiency, satisfactory productivity, simplicity in technical realization, and relatively low price. The main obstacle for the further development of this method is the insufficient study of the processes running in the subsurface of laser target under the ablation of impulses of different duration. The main principles of laser erosion of silver targets by nanosecond pulses which lead to the formation of nanosized metal objects above the zone of irradiation influence are summarized at the 
present work.

\section{Theory}

\section{The character of silver targets destruction under action of nanosecond laser pulses}

The influence of intensive nanosecond optical radiation pulses on the silver target causes the partial absorption of the pulse energy by conduction electrons in the thin subsurface zone [7]. It's important to note that primarily the high reflectivity coefficient of optical radiation by silver surfaces ( $\mathrm{R}=0.97$ for $\Omega=1064 \mathrm{~nm}[8]$ ) may drastically drop if exposed intensively by laser and, as a result, may increase the integrated dose of the absorbed energy to $50 \%$ of the optical energy that reached the surface of the target [9]. Considering that the duration of the leading edge of pulse is less than the thermal relaxation time for silver, the excess energy has no time to penetrate deep into the target at the expense of thermal conductivity. Thus a macro-layer of the change of phase 'solid-vapour' is formed in the thin subsurface target layer omitting the liquid phase state under the influence of the absorbed optical power [10]. Upon that the radiant energy is spent through two main channels: the elevation of inner energy of the formed plasma and the increasing of potential energy of target portion which is compressed by inward-spreading impulse wave. The similar model of laser erosion is referred to as hydrodynamic as the main principles of interaction are determined by hydrodynamic motion of plasma [11].

Under the duration of the leading edge of pulse intensity equal $\sim 50 \mathrm{~ns}$ the initiation of plasma starts with the delay of $\sim 30-40$ ns relative to the beginning of the influence which indicates that light energy of nearly whole leading edge of pulse reaches the target surface without obstruction. From then on the newly formed plasma plume starts absorbing the energy of the falling edge of the acting laser pulse. So the plasma formation increases its inner energy and spreads perpendicularly to the target surface. After the fall of laser pulse intensity the adiabatic plasma spread goes on which leads to its gradual cooling. In this respect, the local fluctuations of density are formed primarily in the plasma plume. Subsequently those fluctuations turn into the solid particles of target material through the behavior of condensation processes. The average size of those particles fall in the nanosized range of 40-50 ns depending on the condition of influence. This condensed phase of silver in the form of nanoparticles occurs in the subsurface target zone quite a long time: during 500-600 Ms after the influence [12]. The described process of laser erosion is accompanied by the appearance of typical terrain change in the form of meandering micro-channels with the cross dimension of several microns and the longitudinal size of scores of microns.

The undertaken studies showed that the initial relief of the target surface significantly influences the effectiveness of the formation of condensed phase of silver. Sharp roughness in the form of longitudinal scratches $\sim 50-100 \mu \mathrm{m}$ on the target surface significantly increases the concentration of nanoparticles in the erosive jet as compared with the usage of the smooth targets with the roughness of $\sim 1-3 \mu \mathrm{m}$. This effect can be explained by increasing of the effective area of interference in rough targets as well as by the occurrence of a great number of gas production centres. The effect of decreasing of silver nanoparticles concentration in the erosive torch for each of the following pulses was found out during the examination of target terrain influence on the effectiveness of nanoparticles laser fusion under the repeated laser action without changing the focusing spot. Four or five similar exposures showed that the effectiveness of nanoparticles formation is the same as in smooth targets regardless of target roughness initial level. This principle may be explained by the process of roughness planarization using the series of laser pulses [12]. Other considerations must be taken of this experimental fact for increasing the effectiveness of laser fusion of silver nanoparticles.

Table 1 shows the typical value of size and particles concentration in the condensed phase of silver in erosive laser jet under the influence of intensive laser pulses on rough targets and on some thermophysical silver characteristics. 


\section{Experimental results and discussion}

\section{Laser synthesis of silver colloidal solutions at water.}

As the table shows a considerable amount $(1012-1013 \mathrm{~cm}-3)$ of particles of the condensed phase of target material is formed in the erosive laser jet of silver targets under the influence of intensive laser pulses. Those particles are typically of the sizes range of 30-40 $\mathrm{nm}$ without the bigger particles of micron range.

It's possible to penetrate the derived particles into the liquid fluid and form by this means the colloidal solution of metal in process. As a rule a small amount of particles is formed under the single exposure of the laser pulse with the examined characteristics. The total particles mass in the condensed phase under the single pulse makes up dozens of micrograms depending on the metal type and the conditions of exposure. For this reason to get more concentrated metal dispersions it's reasonable to use the consecution of laser pulses, which is easily reached with the use of advanced laser technique in frequency mode. The high frequency (up to tens of $\mathrm{Hz}$ ) of the pulse movement makes it possible to make the laser erosion technology effective and competitive comparing to other ways of production of metal dispersions, like chemical metal salt solutions reduction, conductor explosion in liquid, etc. During the synthesis process the constant changing the focusing spot on the target surface must be provided.

The Nd:YAG $(\Omega=1064 \mathrm{~nm})$ laser with the pulse width of $20 \mathrm{~ns}$ and the medium pulse energy of $200 \mathrm{~mJ}$ was used at the present work. Those characteristics allowed getting the densities with the capacity $\sim 1 \mathrm{GW} / \mathrm{cm} 2$ by focusing the spot with $\mathrm{d}=1 \mathrm{~mm}$. The pulse repetition cycle was $5 \mathrm{~Hz}$. The characteristic time of exposure for getting optically solid samples of silver dispersion was set as 10-20 min for each of the samples with volume of $120 \mathrm{ml}$.

The transmission electronic microscopy and absorption spectroscopy (Figure1) were used for the research of silver nanoparticles characteristics in dispersion. The TEM image shows (Figure 1a) that the sample contains nanosized particles of medium size of $\sim 42 \mathrm{~nm}$. The micro-electron diffraction pattern of the sample (Figure $1 \mathrm{~b}$ ) proves that the substance of those particles corresponds with the target substance, i.e. silver.

The spectroscopy (Figure 1c) indicates the occurrence of the typical high of the Surface Plasmon Resonance (SPR) in the sample absorption spectrum. Its maximum falls at $401 \mathrm{~nm}$ which indicates the occurrence of nanoparticles of medium size of about $50 \mathrm{~nm}$ in the colloidal solution [14]. The sample optical dense indicates the particles concentration as of $1011-1012 \mathrm{~cm}-3$.

\section{Laser synthesis of polymer films containing silver nanoparticles}

When using film-forming substances solutions in the capacity of the deposition medium the formation of polymer films containing silver nanoparticles is possible. At the present work under the above-mentioned conditions silver nanoparticles water solutions containing weak polyvinyl alcohol $(\sim 1 \%)$ were formed. From then on, the homogeneous films of dissimilar thickness and geometrical shape were formed from PVA-containing samples by water evaporation at a temperature of $<50^{\circ} \mathrm{C}$. The results of absorption spectroscopy of films of dissimilar thickness are cited in figure 2.

Expectedly, the samples with greater thickness possess the growing optical dense which allows to form modified polymer films with transmission characteristics assigned in advance. It's important to note that the SPR zone which is typical for silver is visibly shifted towards the red area and its maximum forms now $420 \mathrm{~nm}$. This is caused by truly other type of silver particles surrounding which is polymeric matrix. It leads to the noticeable electron density redistribution on the line between nanoparticles and polymeric matrix. The particles images are cited in figure $2 \mathrm{~b}$. They correspond to the study of one sample in various scales. The evenly distributed nanosized silver particles of average size of $\sim 50 \mathrm{~nm}$ may be viewed on the photos [15]. 


\section{Conclusion}

On the basis of the laser fusion method the industrial technology of nanosized particles stream formation may be developed. The main advantages of this technology are the simplicity of technical implementation and the low price of the manufacture. When using water as a medium of implementation, the formation of silver dispersion with high rates of optical dense is possible. By implementing the silver nanoparticles into the film- former solutions, the formation of modified polymeric materials with the absorption band in the visible region is possible. The similar films may be used as the luminosity restrictor in various optical devices. Because of the similarity of the laser erosion processes for different metals the methodology which was worked out will make it possible to form the solutions and the polymer films which contain the nanoparticles of other metallic materials.

\section{References:}

1. Parkansky, N. Scale-up in particle processing / N. Parkansky, B. Alterkop, R. L. Boxman // Powder Technology. - 2005. - Vol. 150, № 36. - P. 91-95.

2. J. Garcia-Barrasa, J. M. Lopez-de-Lusuriaga, M. Monge Silver nanoparticles: sythethis through chemical methods in solution and biometrical applications // Cent. Eur. J. Chem. - 2010.

3. Y. Sun, Ch. An Shaped gold and silver nanoparticles. // Front. Mater. Sci. p. 1-24.

4. D.V. Geohegan, A.A. Puretzky, G. Duscher, S.J. Pennycook Time-resolved imaging of gas phase nanoparticle synthesis by laser ablation // Appl. Phys. Lett. - 1998. Vol. 72, N 3. p. 2987-2989.

5. K. Patel, S. Kapoor, D. P. Dave, T. Mukherjee Synthesis of $\mathrm{Au}, \mathrm{Au} / \mathrm{Ag}, \mathrm{Au} / \mathrm{Pt}$ and $\mathrm{Au} / \mathrm{Pd}$ nanoparticles using the microwave-polyol method //Res. Chem. Intermed. - 2006. Vol. 32, N 2. p. 103-113.

6. V.K. Goncharov, D.R. Ismailov, K.V. Kozadayev, M.V. Pusyrev. Optical behavior of some nanosized structures formed due to laser irradiation Optical memory \& Neural Networks (Information Optics),4 (2007) 254-262.

7. J.F. Ready, D.F. Farson LIA handbook of laser materials processing - Laser Institute of America, 2001. $-715 \mathrm{p}$.

8. Palik, E. Handbook of optical constants of solids / E. Palik. - I ed. - Cambr.: Acad. Press, 1998. - 603 p.

9. A. Evtushenko, E. Ivanik and K. Roџniakowski One Method of Determination of the Effective Absorption Coefficient in Pulsed Laser Irradiation of Metals Journal of Engineering Physics and Thermophysics, 2003, Volume 76, Number 5, Pages 962-968.

10. S.I. Anisimov, B.S. Luk'yanchuk Selected problems of laser ablation theory // Uspehi fizicheskih nauk, . 2003. Vol. 172, N 3. p. 301-333

11. A. Acrivos, T. Tailor Phys. Fluids 5,387, 1962.

12. V. K. Goncharov and K. V. Kozadaev Formation of the condensed phase of metals exposed to submicrosecond laser pulses // Journal of Engineering Physics and Thermophysics, 2010, Volume 83, Number 1, P.90-95.

13. Ahrens T.J. Global Earth physics. A handbook of physical constants. - AGU, 1995. - 376 p. 
14. V. K. Goncharov, K. V. Kozadaev, V. I. Popechits and M. V. Puzyrev Combination method for monitoring the characteristics of aqueous suspensions of metallic nanoparticles // Journal of Applied Spectroscopy, 2008, Volume 75, Number 6, Pages 892-897.

15. V. K. Goncharov, K. V. Kozadaev and D. I. Shiman Formation and complex investigation of spectral-morphologic parameters of Ag nanoscale phase in polymer film // Journal of Applied Spectroscopy, 2010, Volume 77, Number 5, Pages 675-679. 